\title{
Quimioebolização transarterial hepática: modelo experimental de tumor em ratos $^{1}$
}

\author{
Transarterial hepatic chemoembolization: an experimental model in rats
}

\author{
Jamil M. Zarur², Marjorie S. Façanha Barreto ${ }^{3}$,Clarissa Alencar Diogenes ${ }^{3}$, Germana Lopes do \\ Nascimento ${ }^{3}$, Manoel Odorico Moraes ${ }^{4}$
}

1. Trabalho realizado no Laboratório de Oncologia Experimental (LOE) do Departamento de Fisiologia e Farmacologia da Universidade Federal do Ceará (UFC).

2. Cirurgião, Mestrando em Cirurgia na UFC.

3. Aluna do Curso de Medicina de UFC.

4. Doutor em Farmacologia, Professor Adjunto de Fisiologia e Farmacologia da Faculdade de Medicina da UFC, Professor da Pós-graduação do Departamento de Cirurgia da UFC.

\section{RESUMO}

OBJETIVO: Estabelecer um modelo de tumor no fígado de ratos para estudo do comportamento tumoral e avaliar o uso da quimiembolização transarterial .

MÉTODOS: Utilizou-se oitenta e oito ratos Wistar, fêmeas, adultos, pesando entre 175- 284 g . Realizado incisão abdominal de 3 cm e implantou-se o carcinossarcoma de Walker 256 no lóbulo esquerdo do fígado. Dividiu-se em três grupos que receberam respectivamente 100x $10^{3}$, 200x $10^{3}$ e 300x $10^{3}$ células tumorais, avaliado a pega do tumor e a sobrevida. Em outro grupo de experimento com 39 animais inoculados com tumor de Walker foi avaliado a sobrevida dos animais após infusão do 5-Flourouracil (5-FU) por via intraperitoneal e intra-arterial.

RESULTADOS: O implante do carcinossarcoma de Walker no fígado de ratos apresentou desenvolvimento de $100 \%$, teve um crescimento rápido e desenvolvimento de metástases tardiamente, levando os animais ao óbito entre o sétimo e décimo quinto dia. A quimiembolização transarterial é possível de ser realizada experimentalmente. O uso do 5-FU aumentou a sobrevida em comparação ao grupo controle.

CONCLUSÃO: O modelo de implante do tumor de Walker no fígado de ratos é eficiente, de fácil reprodutibilidade, e sobrevida média de 9,96 \pm 0.3 dias. A quimioterapia transarterial hepática pode ser realizada experimentalmente para avaliar diversas drogas.

DESCRITORES: Modelo tumor hepático. Carcinossarcoma de Walker. Quimioembolização.

\section{ABSTRACT}

PURPOSE: An animal model to study the behaviour of liver tumor in rat and its response after use of transarterial chemoembolization.

METHODS: We use 88 Wistar rats, all of them were females, adult, weight 175-284 g. Abdominal incison of three $\mathrm{cm}$ and implanted the Walker carcinossarcoma 256 at left lobule of the liver. The animals were divided into three grups, that received $100 \times 10^{3}, 200 \times 10^{3}$, and $300 \times 10^{3}$ cells. Followed up the animas to avaliate life standing and tumoral development. In another experiment was used 39 animals which already had Walker 256 tumor and we study the survival of the animals after treatment with 5-FU IP or 5-FU IA. 
RESULTS: The orevall tumor development rate were $100 \%$. Tumor growth was fast, and devolopment metastases on old fase. The animals dead between $7^{\circ}$ and $15^{\circ}$ day. Its possible to do chemoembolization experimentaly, after the use of 5-FU the rate survival increased.

CONCLUSION: The model with Walker 256 tumor developed here is easy to repoduce, efficient, with high tumor development rate observed, the life standing is about 9,96 $\pm 0,3$ days. The chemoembolization experiment allows to assess several drugs.

KEY WORDS: Liver tumor model. Walker’s carcinosarcoma. Chemoembolization.

\section{Introdução}

Os tumores primários do fígado e metastáticos apresentam prognósticos ruins, não existindo uma terapêutica eficaz quando em estágios avançados, assim a busca de novas modalidades de tratamento se faz necessária. O estudo experimental é uma forma de testar novas drogas e formas de tratamento.

Idealizamos um modelo experimental utilizando ratos, que foram inoculados com o carcinossarcoma 256 de Walker em diferentes quantidades de células, acompanhado seu desenvolvimento, evolução e sobrevida.

Conhecida a evolução natural do tumor, comparamos o uso do 5-Flourouracil (5-FU) administrado por via intraperitoneal e por via intraarterial, na artéria hepática, juntamente com partículas de polivinil alcool ( PVA) de 45 a 150 microns.

A quimioembolização tem sido utilizada para o tratamento dos tumores hepáticos em estágios avançados, porém seus efeitos, complicações e qual droga apresenta benefícios ainda não são conhecidas.

\section{Métodos}

O tumor de Walker vem sendo mantido no Laboratório de Oncologia Experimental da Universidade Federal do Ceará através de transplantes sucessivos a cada 8 dias, por inoculação via intramuscular na pata traseira de ratos Wistar. O tumor é retirado dos animais doadores e colocado em uma placa de Petri contendo $9 \mathrm{ml}$ de Ringer com lactato e $1 \mathrm{ml}$ de gentamicina ( $10 \mathrm{mg} / \mathrm{ml})$. O tecido é separado de focos necróticos e triturado em pequenos fragmentos, em seguida colocados em um Erlenmeyer estéril e submetidos a um agitador com bala magnética por 5 minutos. A solução é então filtrada e colhido $10^{6}$ células tumorais para nova manutenção do tumor ou experimentos .

Para inoculação no fígado de ratos, foi utilizado ratos Wistar, fêmeas com peso entre 175 a 284 g. A pesquisa foi aprovada pela comissão de ética de experimentos em animais.

Realizado incisão mediana abdominal de $3 \mathrm{~cm}$, estando os animais sob anestesia com hidrato de coral a $10 \%$ na dose $0,1 \mathrm{ml} / 30$ gamas de peso por via intraperitoneal . Uma solução de $0,1 \mathrm{ml}$ com o tumor de Walker era inoculada utilizando-se uma seringa de $1 \mathrm{ml}$ e agulha 30 G1/2 sob visão direta no lóbulo esquerdo do fígado. Após a inoculação a parede abdominal era suturada com mononylon 5.0 em plano único e mantidos em observação até sua recuperação anestésica e posteriormente mantidos no biotério até sua morte ou sacrifício (Figura 1).

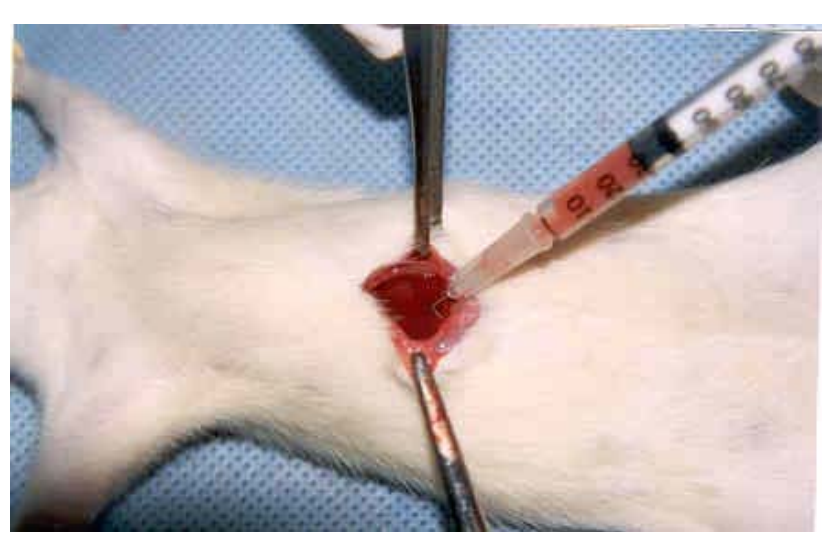

FIGURA 1 - Implante tumoral.

Para realização do trabalho separamos em grupos de experimentos. O primeiro experimento teve a finalidade de estabelecer a quantidade de células que seriam necessárias para o desenvolvimento do tumor. Para tanto, três animais foram inoculados com $100 \times 10^{3}$ células do tumor, três animais foram inoculados com 200×103 células e um terceiro grupo de três animais foram inoculados com 
300x10 $0^{3}$ células do tumor de Walker, os animais eram sacrificados no $6^{\circ}$ dia e analisados macroscópicos e microscopicamente.

No segundo experimento os animais foram inoculados com $100 \times 10^{3}$ células (grupo A1), 200x10 células (grupo A2) e $300 \times 10^{3}$ células (grupo A3) do tumor de Walker e observados até a sua morte espontânea, esse grupo teve a finalidade de estudar a sobrevida dos animais. Após a morte espontânea realizava-se a necropsia com inventário da cavidade abdominal e torácica retirada do fígado com o tumor para estudo de microscopia, realizava-se ainda as medidas do tumor com uso de um paquímetro para cálculo do volume tumoral. Foram utilizados um total de quarenta ratos Wistar, distribuídos em treze no grupo A1, quinze no grupo A2, e doze no grupo A3.

Em um terceiro experimento os animais foram inoculados com $100 \times 10^{3}$ células do tumor de Walker e divididos em três grupos. No grupo B1, grupo controle, no $6^{\circ}$. dia após a inoculação os animais eram submetidos a novo procedimento cirúrgico com abertura da cavidade peritoneal, no pedículo hepático identificava-se e cateterizava-se a artéria hepática com cateter de 0,85mm de diâmetro (Figuras 2 e 3), era então infundido $1 \mathrm{ml}$ de solução salina, após realizavase sutura do orifício de entrada do cateter com fio cirúrgico prolene 7.0 e fechamento da cavidade abdominal. O grupo B2, no $6^{\circ}$. dia após a inoculação administrava-se 5-Fluorouracil intra-peritoneal da dose de $130 \mathrm{mg} / \mathrm{massa}$ corporal $\left(\mathrm{m}^{2}\right)$. No grupo B3 , também no $6^{\circ}$. dia após a inoculação os animais eram submetidos a novo procedimento cirúrgico com abertura da cavidade peritoneal, identificação e cateterização da artéria hepática e infundia-se 5Fluorouracil intra-arterial na dose anteriormente descrita juntamente com 200mg/kg de Polivinil alcool ( ContourÒ, Boston Cientific , USA) (diâmetro de 45-150 microns). Os animais eram então mantidos em observação até sua morte, foram utilizados neste terceiro experimento trinta e nove animais, distribuídos em 12 no grupo B1, 12 no grupo B2 e 15 no grupo B3.

Realizado análise estatística pelo método de Kaplan-Meier e a diferença de sobrevida entre os grupos pelo teste de Log Rank, sendo os valores de $\mathrm{p}<0,05$ considerados significantes.

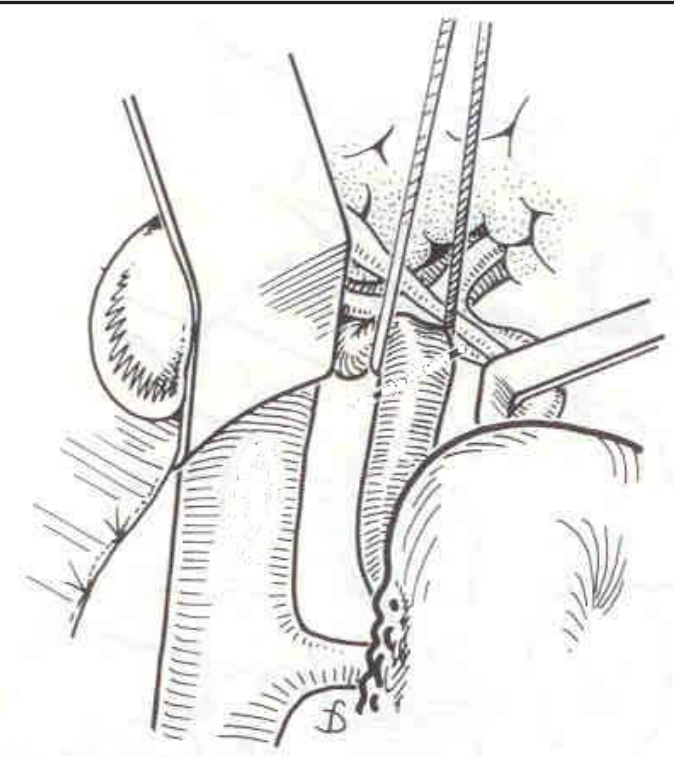

FIGURA 2 - Artéria hepática.

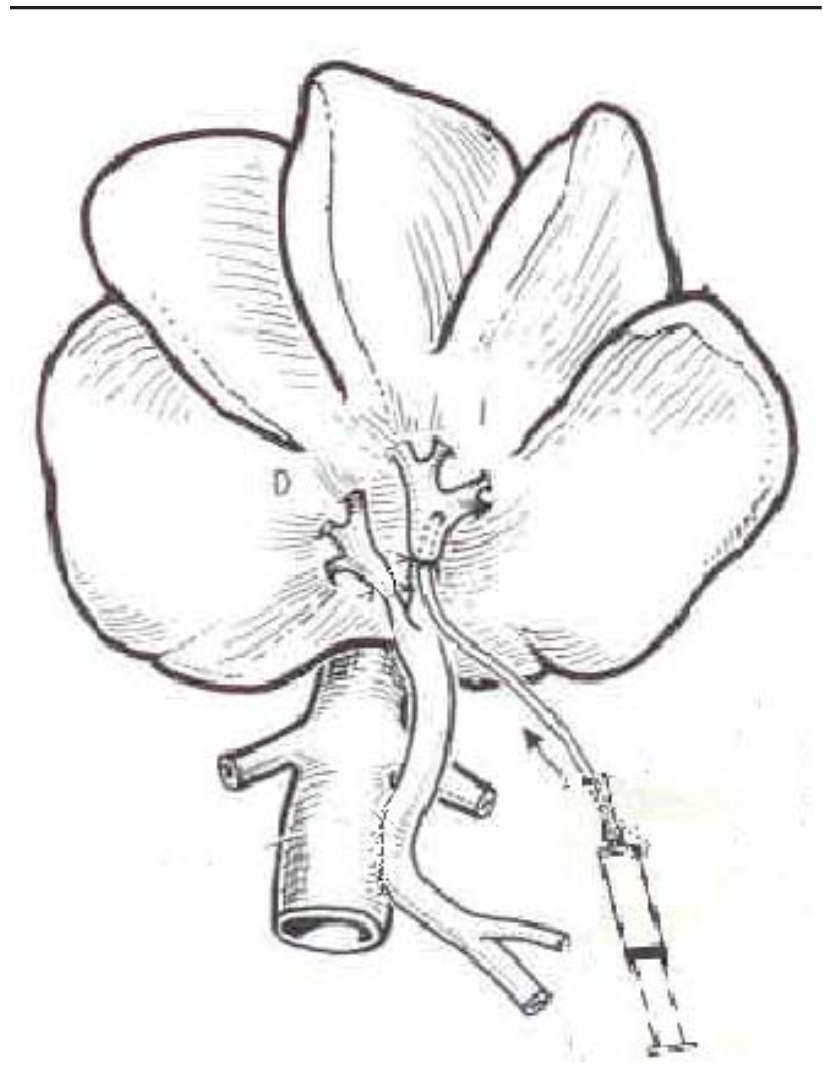

FIGURA 3 - Infusão intra-hepática.

\section{Resultados}

No primeiro experimento após o sacrifício dos animais no $6^{\circ}$ dia foi encontrado o tumor de Walker no fígado de todos os ratos. A pega do tumor foi de $100 \%$. Formavam massa tumoral no local do implante, de cor clara e consistência endurecida, com cerca de 0,5 cm de diâmetro, havia invasão de 
estruturas contíguas em alguns casos, porém não encontramos implantes na cavidade abdominal ou em outros órgãos. À microscopia encontramos células tumorais do carcinossarcoma 256 de Walker agrupadas, em algumas amostras as células tumorais estavam presentes no sinusóides hepáticos (Figura 4).

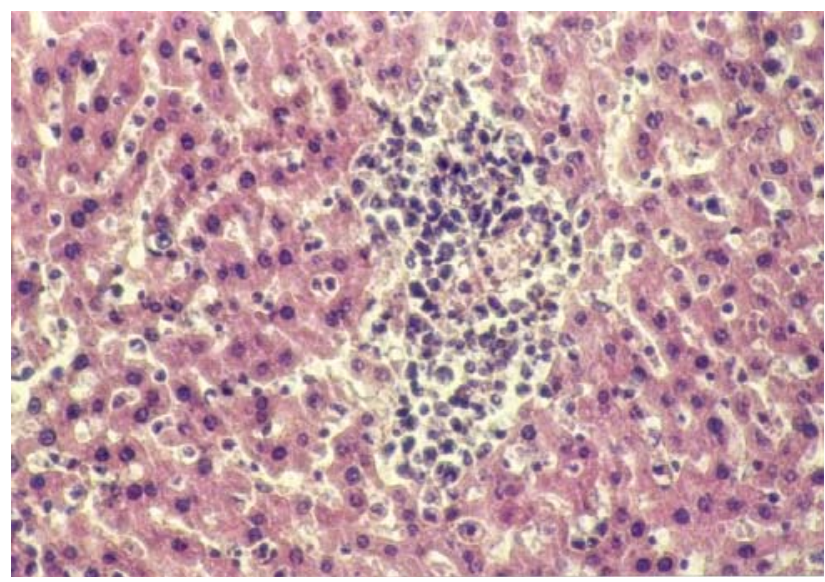

FIGURA 4 - Microscopia - Tumor de Walker no fígado.

No segundo experimento os animais foram a óbito entre o sétimo e o décimo quinto dia. No grupo A1 os animais tiveram óbito entre o $9^{\circ}$ e o $15^{\circ}$ dia, a sobrevida média foi de 10,23 dias . No grupo A2 as mortes ocorreram entre o $9^{\circ}$ e $11^{\circ}$ dia, a sobrevida média foi de 9,73 dias . No grupo A3 os óbitos ocorreram entre o $7^{\circ}$ e $14^{\circ}$ dia, com sobrevida média de 10,0 dias. Não ocorrendo diferença estatística da sobrevida entre os grupos(Figura 5). O volume

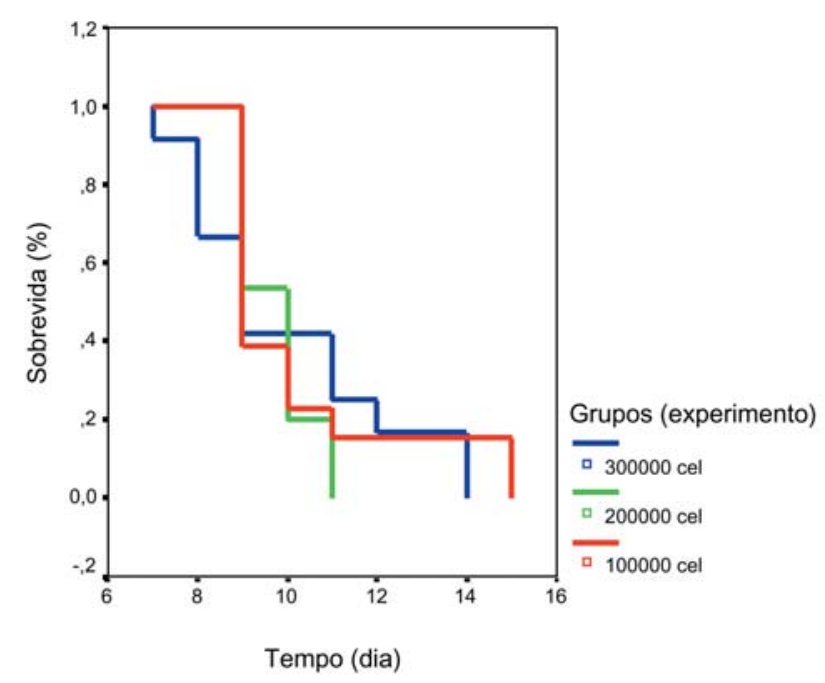

FIGURA 5 - Gráfico de sobrevida no segundo experimento. do tumor variou de $0,016 \mathrm{~cm}^{3}$ a $0,426 \mathrm{~cm}^{3} \mathrm{com}$ média de $0,173 \mathrm{~cm}^{3}$.

No terceiro experimento os animais que tiveram morte decorrentes do procedimento cirúrgico foram retirados do experimento. $\mathrm{O}$ grupo controle ( grupo B1 ) teve uma sobrevida média de 10,58 dias, os animais tiveram óbito entre o $9^{\circ}$ e o $11^{\circ}$ dia. O grupo B2 com infusão de 5-FU intraperitoneal teve sobrevida média de 11,9 dias, as mortes ocorreram entre o $10^{\circ}$ e $14^{\circ}$ dia e no grupo B3 com infusão intraarterial a sobrevida média foi de 12,27 dias os animais tiveram óbito entre o $11^{\circ}$ e o $15^{\circ}$ dia (Figura 6).

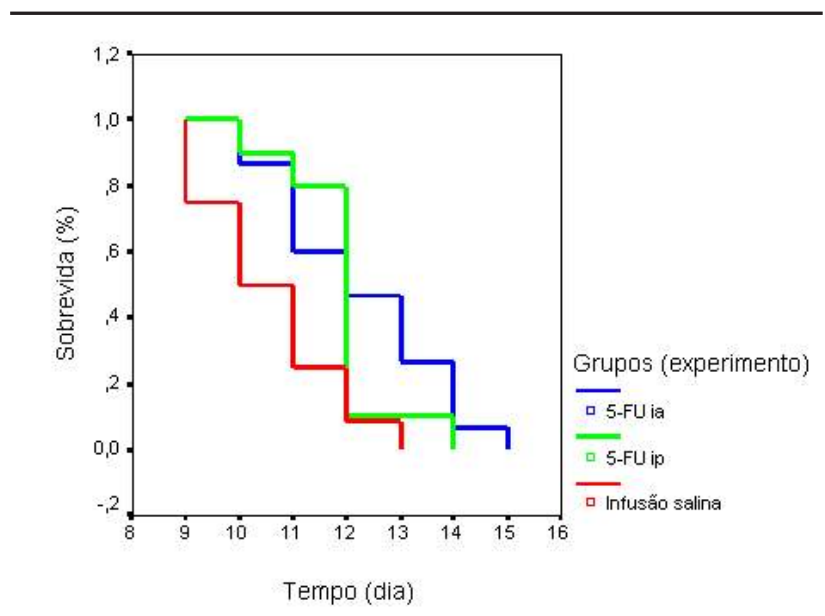

FIGURA 6 - Gráfico de sobrevida no terceiro experimento.

A analise estatística pelo de método de KapanMeier com análise da sobrevida pelo teste de $\log$ Rank mostrou significância estatística entre o grupo controle e o grupo com infusão de 5-FU por via peritoneal $(p<0,020)$ e entre o grupo controle e o grupo com infusão intra-arterial $(p<0,063)$. Entre o grupo B2 e B3 não houve diferença estatística ( $p$ $=0,44)$

\section{Discussão}

Modelos experimentais de tumores no fígado de ratos já foram desenvolvidos através da inoculação do tumor no baço e em veias do sistema mesentérico que drenaram através da veia porta para o fígado, porém apresentam desenvolvimento tumoral variável e com baixa mortalidade ${ }^{1,2}$.

A indução de lesões cancerígenas no fígado de ratos também foi desenvolvido com o uso de $\mathrm{N}$ nitrosodiethylamine ${ }^{3}$, p-dimetil-aminoazobenzene, 2acetilaminofluorene, e 2-diacetilaminofluorene por via 
oral através da dieta durante 4 a 6 meses $^{4}$.

A indução de tumor no fígado de ratos através da inoculação direta foi utilizado com vários tipos de células tumorais, como o de Novikoff, o adenocarcinoma do colon CC531 ${ }^{5}$ e células tumorais de Lewis ${ }^{6}$. O tumor de Walker foi utilizado como modelo pelo implante direto no fígado de ratos, o modelo mostrou ser reprodutível com elevada taxa de desenvolvimento e características de crescimento local.Apresenta suprimento sangüíneo principalmente pela artéria hepática, com hipervacularização, similar como o $\mathrm{HCC}^{7}$

O uso de tumor VX2 no coelho através do implante direto ou na veia mesentérica é um modelo bem conhecido e bastante utilizado para avaliar meios de diagnósticos de imagem e ação terapêutica de medicamentos ${ }^{8}$.

O uso do tumor carcinossarcoma de Walker vem sendo utilizado no laboratório de Cirurgia experimental da Universidade Federal do Ceará para inoculação em diversos órgãos de ratos, como estômago, pulmão e rins ${ }^{9,10,11}$.

O implante do tumor de Walker no fígado de ratos através da inoculação direta demostrou ser uma técnica fácil, e pega de 100\%. Demostra ser um tumor agressivo com crescimento no parênquima hepático e invasão por contiguidade, a taxa de sobrevida é pequena e sem diferenças significativas com as diferentes quantidades de células implantadas e por nós utilizados. O procedimento de infusão de drogas na artéria hepática requer conhecimento da anatomia e material cirúrgico adequado. A via de administração intra-arterial não alterou a sobrevida com o uso do 5-FU no presente trabalho.

Modelos animais de tumor hepático vêem sendo utilizados para o desenvolvimento e aprimoramento de meios diagnósticos por imagem ${ }^{12,13,14,15}$ e para testes de novas terapias farmacológicas ${ }^{16,17}$, cirúrgicas e meios físicos de hipertermia e hipotermia.

A quimioembolização tem sido considerado como efetivo no tratamento do carcinoma hepatocelular (HCC) em pacientes que não são candidatos a cirurgia. Mas grandes variações no método empregado e nas drogas utilizadas diferem bastante nos estudos já realizados. A reprodução de um modelo animal para realizar quimioembolização irá ajudar no conhecimento desta nova técnica, estudando as diferentes estratégias terapêuticas e avaliando as reações adversas relacionadas com a quimioembolização transarterial.

\section{Conclusão}

O modelo de implante de tumor carcinossarcoma de Walker em fígado de ratos Wistar provou ser bastante eficaz, o procedimento de implantação é simples e rápido, o tumor tem crescimento rápido e com sobrevida média de $9,96 \pm 0,3$ dias. Demonstra ter comportamento tumoral restrito ao fígado formando massa tumoral de 0,173 $\mathrm{cm}^{3}$. O procedimento de cateterização da artéria hepática é possível e requer experiência. O modelo experimental pode ser assim utilizado para linhas de pesquisa que avaliem drogas quimioterápicas por via transarterial.

\section{Referências}

1. Lafreniere R, Rosenberg S. A novel approach to the generation and identification of experimental hepatic metastases in a murine model. : J Natl Cancer Inst 1986 Feb;76(2):309-22.

2. Yang R., Rescorla F.J., Reilly C.R., Faught P.R., Sanghvi N.T., Lumeng L., Franklin T.D., Grosfeld J.L. A reproducible rat liver cancer model for experimental therapy: introducing a technique of intrahepatic tumor implantation. J Surg Res 1992; 52: 193-98 .

3. Goldrosen M.H., Paolini N. Jr, Holyoke E.D. Description of a murine model of experimental hepatic metastases. J Natl Cancer Inst 1986;77(3):823-8 .

4. Port, G. D. Inductionof tumors by chemicals. Biol Cel 1999;02(2): 76-81.

5. Kopper L, Van Hanh T, Lapis K Experimental model for liver metastasis formation using Lewis lung tumor. Cancer Res Clin Oncol 1982;103(1):31-8.

6. Thomas C, Nijenhuis AM, Timens W, Kuppen PJ, Daemen T, Scherphof GL. Liver metastasis model of colon cancer in the rat: immunohistochemical characterization. Inv Metast 1993;13(2):102-12.

7. Li X., Zheng C., Feng G., Zhuo C., Zhao J., Liu X. An implantable rat liver tumor model for experimental transarterial chemoembolization therapy and its imaging features . World J Gastroenterol 2002;8(6):1035-9.

8. Geschwind, J.H., Artemov, D., Abraham, S., Omdal, D., Huncharek, M., McGee, C., Arepally, A., Lambert, D., Venbrux, A.C., Lund, G. B. Chemoembolization of liver tumor in a rabbitmodel: assessment of tumor cell death with diffusion-Weighted MR imaging and histologic analysis. J Vasc Radiol 2000; 11:1245-55.

9. Silva LFG, Soares FSD, Anselmo JNN, Cavalcante JLBG, Moraes MO, Vasconcelos PRL. Modelo de tumor experimental em rim de ratos. Acta Cir. Bras. 2002;17(1): 62-6.

10. Gomes-Neto A, Pessoa BBGP, Aguiar SA, Furtado BM, 
Moraes MO, Ribeiro RA. Modelo de tumor de pulmão em rato com o carcinossarcoma de Walker. Acta Cir Bras 2002;17(1): 12-22.

11. Oliveira, PFM, Henriques IA, Rodrigues Filho F, Almeida, PRC, Moraes MO. Estabelecimento de um modelo de tumor experimental pela inoculação do tumor de Walker em estômago de rato. Acta Cir Bras 1998;13(4):243-48 .

12. Pavone P, Patrizio G, Pietroletti R, Festuccia C, Stark DD, Elizondo G, Passariello R. Induction of liver metastases in the rat. An experimental model for research on magnetic resonance. Radiol Med 1989;77(1-2):94-8.

13. Qin Y, Van Cauteren M, Osteaux M, Willems G. Quantitative study of the growth of experimental hepatic tumors in rats by using magnetic resonance imaging. Int J Cancer 1992;51(4):665-70.

14. Ni Y, Marchal G, van Damme B, van Hecke P, Michiels J,
Zhang X, Yu J, Baert AL. Magnetic resonance imaging, microangiography, and histology in a rat model of primary liver cancer. Invest Radiol 1992;27(9):689-97.

15. Chen MC, Tsang YM, Stark DD, Weissleder R, Saini S, Brandhorst J, White DL, Engelstad BL, Ferrucci JT . 8 Hepatic metastases: rat models for imaging research . Magn Reson Imaging 1989;7(1):1.

16. Kurth S, Bulian D, Kreft B, Riemenschneider T. Intraarterial hepatic chemotherapy with fluorouracil, fluorodeoxyuridine, mitomycin $\mathrm{C}$, cisplatin or methotrexate as single-agent anticancer drugs for a transplanted experimental liver tumor in rats. J Cancer Res Clin Oncol 1996;122(7):421-6.

17. Nagel JD, Kort WJ. A new liver-tumor model in the rat. Cancer Chemother Pharmacol 1992;30(1):70-2.

Correspondência:

Jamil M. Zarur

Rua Silva Paulet, 2020/1303

60120-240 Fortaleza-CE

jamilzarur@uol.com.br

Recebimento: 03/06/2004

Revisão: 10/07/2004

Aprovação: 28/08/2004

Conflito de interesse: nenhum

Fonte de financiamento: nenhuma

\section{Como citar este artigo:}

Zarur JM, Barreto MSF, Diógenes CA, Nacimento GL, Moraes MO. Quimioembolização transarterial hepática um modelo experimental de tumor em ratos. Acta Cir Brás [serial online] 2004 Set-Out;19(5). Disponível em URL: http://www.scielo.br/acb [também em CD-ROM].

* Figuras coloridas disponíveis em $\underline{w w w . s c i e l o . b r / a c b}$ 